\title{
A cluster of cholera among patients in a Vietnamese district hospital in 2010
}

\author{
Thuan Huu Vo ${ }^{1,2}$, Ninh Hoang Le ${ }^{1}$, J. Pekka Nuorti ${ }^{3}$, Lan Trong Phan ${ }^{2}$, Nguyen Nhu Tran Minh ${ }^{2}$ \\ ${ }^{1}$ Department of Planning and Technical Support, Institute of Hygiene and Public Health, Ho Chi Minh City, Vietnam \\ 2 Vietnam Field Epidemiology Training Program, Hanoi, Vietnam \\ ${ }^{3}$ School of Health Sciences, University of Tampere, FI-33014 Tampere, Finland
}

\begin{abstract}
On July 20, 2010, three cases of cholera were reported from a district hospital in Ca Mau province, Vietnam. We investigated the likely source and mode of transmission of the outbreak. All hospitals in the province were requested to notify cases of acute watery diarrhoea. Epidemiological, clinical, and laboratory data were collected. Between July 12 and 22, seven cases with positive culture for Vibrio cholera were identified. Six cases were epidemiologically linked to the index case. Basic infection control practices were not in place at the hospital. Clinicians and public health staff should consider the possibility of nosocomial cholera transmission even in non-endemic areas.
\end{abstract}

Key words: Cholera; outbreak; diarrhoea; infection control

J Infect Dev Ctries 2013; 7(12):910-913. doi:10.3855/jidc.3503

(Received 05 March 2013 - Accepted 09 June 2013)

Copyright $(92013$ Vo et al. This is an open-access article distributed under the Creative Commons Attribution License, which permits unrestricted use, distribution, and reproduction in any medium, provided the original work is properly cited.

\section{Introduction}

Cholera re-emerged in the northern provinces of Vietnam in 2007. It spread throughout the country including the southern provinces, a lowland region with entangled canals. On July 20, 2010, a district hospital reported three cases of cholera to $\mathrm{Ca} \mathrm{Mau}$ Provincial Preventive Medicine Centre (PMC). Cholera had not been reported in the province for the past 10 years. As local residents generally use water from canals around their homes and dispose human waste into these canals, public health officials were concerned about the potential risk of high morbidity and mortality in the population. We conducted an investigation on July 22, 2010, to identify the likely source and mode of transmission of the outbreak.

\section{Methodology}

According to provincial health statistics in 2010, the population of the district was 88,461 , with 19,150 households; the proportion using safe drinking water was estimated to be $20 \%$ to $30 \%$.

A case was defined as a resident of the district of any age with acute watery diarrhoea and positive culture for Vibrio cholera between July 12 and 22, 2010. Following the initial report, all hospitals in the province were requested to notify the PMC of any cases of acute watery diarrhoea. Faecal specimens were sent to provincial and regional laboratories for culture. Cultures were obtained using Thiosulphate, citrate, bile salts, and sucrose agar. Patients or parents of children meeting the case definition were interviewed at the hospital within one week of illness onset. A standard questionnaire was used in the interviews. The patients or the children's parents were shown calendars to help them recall the dates that specific food items were consumed. Information obtained from hospital records and interviews of patients and their parents included demographics; clinical symptoms; food items consumed in the three days before the onset of diarrhoea; water sources used for drinking and other activities; knowledge and practices on diarrhoea disease prevention; and personal and food hygiene practices. Details of clinical symptoms, signs, diagnoses, and treatments were transcribed from hospital records. Water samples were taken from the hospital and from patients' households.

\section{Results}

Between July 12 and 22, seven cholera cases were identified through hospital investigations. Six cases were from the same village; five were males whose ages ranged from seven days to 72 years; the median 
Figure 1: Culture-confirmed cholera patients by date of onset. Julv 2010. Ca Mau. Vietnam

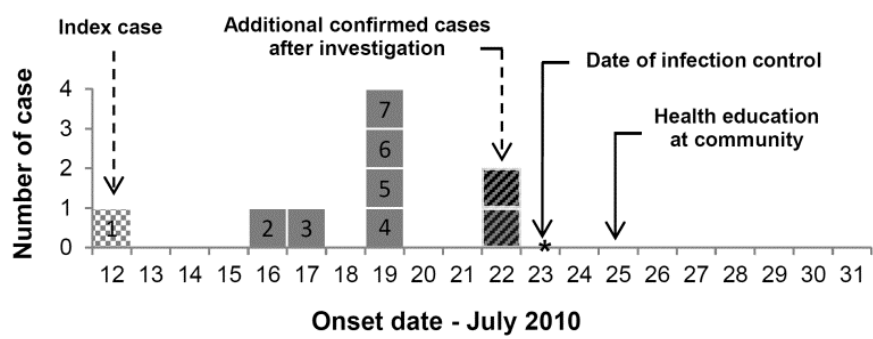

*No more cases identified by surveillance until 21 days from the last case

age was two years. All cases had a positive culture for Vibrio cholera O1, biotype El Tor, serotype Ogawa. Figure 1 shows the culture-confirmed cases by date of illness onset. Four cases were admitted to the hospital between three and 18 hours of the onset of illness. All cases admitted to the hospital had severe watery diarrhoea, and four cases had vomiting. No cases were fatal. Medications for the seven cases included rehydration (Ringer's lactate in seven cases; oral rehydration salts solution in five cases), antibiotics (erythromycin in five cases; cefuroxime in one case; gentamycin in one case), probiotics (four cases), and smectites for intestinal adsorbent (four cases).

The index case had not been identified or treated as a cholera case at the hospital until the laboratory test results were received four days later. Hospital infection control was implemented four days after admission of the index case, but basic infection control practices still did not comply with guidelines of the Ministry of Health (MOH) at the time of investigation [1]. Particularly, the entrance and exit to the isolation area were not restricted; hand-washing bowls, carpet, and gowns were not present in front of the isolation room's door; a chlorine solution to wipe the isolation room's floor was below the required minimum chlorine concentration; door handles were not cleaned; hospital bed sheets were not changed regularly; dirty clothes of patients were left in the corner of the room; and the bathroom in this room was far below the minimum hygiene standards [1].

Six cases were epidemiologically linked to the index case. Their severe watery diarrhoea began four to seven days after that of the index case. Of the six secondary cases, three cases were acquired at the hospital; one was a seven-day-old newborn sharing the hospital room with the index case, two were inpatients in the same ward and shared home-prepared food with the index case, and three were relatives of and shared the same caregivers with the index case. Two additional cases confirmed after the investigation were relatives of case three and case five. They had visited the cases at the hospital. Ciprofloxacin (500 mg x 2) was administered to close contacts of the cases only after the index case had been confirmed. Table 1 shows demographic, clinical, and epidemiologic characteristics of the cholera patients.

No suspected foods items (e.g., seafood, ice cubes, raw vegetables) were identified in the epidemiologic investigation. Drinking water was taken from drilled wells within a 300-metre distance of patients' residences. Water was transported by hand and/or boats in open plastic 10- to 20- litre containers and stored in 50- to 100-litre jars at the households. The water was chlorinated inappropriately, though the water chlorination was guided by health workers. Patients and their family members had not boiled the drinking water before the illnesses occurred. Water for other daily activities was taken from canals, which are directly linked to the residents' water latrines; the latrines were situated above the residents' fish ponds. Also, other waste was thrown into the canals. Caregivers had poor knowledge and practices of personal and food hygiene, no knowledge about hand washing when needed or about preparing, consuming, and storing safe drinking water and foods. The caregivers also had inadequate knowledge about prevention measures for diarrhoeal diseases; they

Table 1: Demographic and epidemiologic data for seven cholera patients in Ca Mau, Viet Nam, 2010

\begin{tabular}{|c|c|c|c|c|c|c|}
\hline Case No. & Age/gender & $\begin{array}{l}\text { Onset date } \\
\text { (2010) }\end{array}$ & $\begin{array}{c}\text { Admission date } \\
\text { (2010) }\end{array}$ & $\begin{array}{l}\text { Contact to the } \\
\text { index case }\end{array}$ & $\begin{array}{c}\text { Shared caregiver } \\
\text { with }\end{array}$ & $\begin{array}{c}\text { Condition of previous } \\
\text { admission }\end{array}$ \\
\hline $2 * *$ & 7 days/female & $16 / 7$ & $09 / 7$ & Yes & - & Delivery \\
\hline 4 & 72 years/male & $19 / 7$ & $06 / 7$ & Yes & - & Gastritis \\
\hline 5 & 1 years $/$ male & $19 / 7$ & $20 / 7$ & No & Case 1 & - \\
\hline 6 & 6 years $/$ male & $19 / 7$ & $10 / 7$ & Yes & Case 2 & Asthma \\
\hline
\end{tabular}

*Case 1 and 5 were brothers; case 3 and 7 were siblings; all four were cousins

**Case 2, 4 and 6 are relatives; case 2 and 6 lived in same house and were cared for by the same grandmother 
believed that chemoprophylaxis and vaccination were the best ways to prevent diarrhoea and did not know that diarrhoea diseases can be prevented by hand washing, consuming thoroughly cooked food items, and safe disposal of human excreta. No cholera was identified from water samples taken at the hospital or patients' households.

\section{Discussion}

Our study identified three main reasons for the spread of cholera at the hospital. First, inappropriate hospital infection control was likely the key failure. Since cholera had not been reported in the province during the past 10 years, health professionals may no longer consider cholera as a possible cause of diarrhoea. Second, educational messages for the population at risk were ineffective in reducing the risk of infection in close contacts. Third, steps for standard outbreak investigations or World Health Organization (WHO) cholera control and prevention guidelines were not followed appropriately at the hospital [2]. Moreover, antibiotics, smectites, or probiotics are not recommended in the guidelines of $\mathrm{MOH}$ [1] or WHO for the treatment of cholera, as the clinical benefit is very small [3].

Standard hospital infection control measures should be implemented immediately at hospitals once cholera is reported in the community [4], even when a single patient is admitted with watery diarrhoea and symptoms compatible with cholera [5]. Close contact with cholera patients may result in infection [6]. Poor hygiene practices are known risk factors [7], so infected caregivers may transmit cholera to children in their households. Although antibiotics may reduce the infection risk among inpatients [8], two inpatients at the hospital did not receive any antibiotics before contracting cholera. Furthermore, health education during outbreaks could be effective in the control of cholera [9]. Health education messages should focus on water treatment and avoiding contamination during distribution and storage of water at home [7].

Although our study focused only in one hospital, the findings are applicable to the control and prevention of cholera elsewhere in Vietnam. Bias was minimized as the investigators were trained and calendars were used to minimize recall bias. However, delayed response was a limitation of the study. In fact, faecal samples of close contacts could not be tested because they had already received chemoprophylaxis. Negative results of cholera in environmental samples could be due to sub-optimal methods for detection of cholera in water environments and chlorinated households' water.

Based on the findings of this study, public health actions were immediately implemented. Extensive control measures were implemented at the hospital, and operational communications in the community on cholera control and prevention were deployed. No new cases were reported after the control measures at the hospital and in the community were implemented.

This cluster of cholera resulted from inappropriate hospital infection control and inadequate personal hygiene and food hygiene practices. No new cases were reported after standard infection control measures were implemented at the hospital, emphasizing the critical role of hospitals in controlling cholera transmission. Clinicians and public health staff should be aware of the possibility of cholera in nonepidemic areas as well, so that case reporting, investigation, and responses can be done as soon as possible. Local health workers should be trained in the principles of field epidemiology, and laboratories should be equipped to collect and test environmental samples.

\section{Acknowledgements}

We thank Provincial Preventive Medicine Centre of Ca Mau for reporting the outbreak and for assistance the investigation. We thank members of the outbreak control team of Institute of Hygiene and Public Health who assisted with data collection.

\section{References}

1. Ministry of Health (2010) Control of Cholera Manual. Hanoi: Hanoi Press $21 \mathrm{p}$.

2. World Health Organization (2008) Foodborne disease outbreaks: guidelines for investigation and control. Geneva: WHO Press 162 p.

3. Cézard JP, Bellaiche M, Viala J, Hugot JP (2007) Medication in infectious acute diarrhea in children. Arch Pediatr 14: S169-175.

4. Swaddiwudhipong W, Peanumlom P (2011) A Case of Nosocomial Cholera during a Community Outbreak in a ThaiMyanmar Border Area. J Med Assoc Thail 93: 1112-1114.

5. Hernández JE, Mejía CR, Cazali IL, Arathoon EG (1996) Nosocomial infection due to Vibrio cholerae in two referral hospitals in Guatemala. Infect Control Hosp Epidemiol 17: 371-372.

6. Tamayo JF, Mosley WH, Alvero MG, Joseph PR, Gomez CZ, Montague T, Dizon JJ, Henderson DA (1965) Studies of cholera El Tor in the Philippines: Transmission of infection among household contacts of cholera patients. Bull WHO 33: 645-649.

7. Kirk MD, Kiedrzynski T, Johnson E, Elymore A, Wainiqolo I (2005) Risk factors for cholera in Pohnpei during an outbreak in 2000: lessons for Pacific Countries and Territories. Pac Health Dialog 12: 17-22. 
8. Ryder R, Rahman A, Alim A, Yunis M, Houda B (1986) An outbreak of nosocomial cholera in a rural Bangladesh hospital. J Hosp Infect 8: 275-282.

9. Quick RE, Gerber ML, Palacios A, Beingolea L, Vargas R, Mujica O, Moreno D, Seminario L, Smithwick EB, Tauxe RV (1996) Using a knowledge, attitudes and practices survey to supplement findings of an outbreak investigation: cholera prevention measures during the 1991 epidemic in Peru. Int $\mathbf{J}$ Epidemiol 25: 872-878.

\section{Corresponding author}

Thuan Huu Vo

159 Hung Phu Street, District 8

Ho Chi Minh City, Vietnam

Phone: (+84) 983237198

Fax: (+84 8) 38563164

E-mail: vo.huuthuan@yahoo.com

Conflict of interests: No conflict of interests is declared. 